\title{
Atención en salud para migrantes: un desafío ético
}

\author{
Atenção na área da saúde para migrantes: um desafio ético \\ Health care for migrants: an ethical challenge
}

\section{Mónica Burgos Moreno', Tatiana Parvic Klijn'}

'Universidad de Concepción. Facultad de Medicina. Departamento de Enfermería. Concepción, Chile.

\author{
Submissão: 06/08/2010 Aprovação: 08/09/2010
}

\section{RESUMEN}

El artículo tiene como propósito evidenciar que el fenómeno migratorio en aumento en la sociedad actual, ha traído consigo oportunidades, pero también dificultades para sus protagonistas. La población migrante corresponde a un grupo vulnerable, especialmente en problemas sanitarios. La atención en salud del migrante representa un reto para la salud pública, no solo considerando políticas de acceso y de información, sino que sobre todo por la atención basada en un trato humanizado respetando las creencias, valores y expectativas. Una atención digna exige un compromiso real de los estados y sensibilidad de los proveedores de atención de los servicios de salud, si se pretende otorgar servicios integrales basados en el respeto de los derechos humanos a esta población.

Palabras clave: migrante, salud, ético.

\section{RESUMO}

O artigo tem como propósito evidenciar que o fenômeno migratório em aumento na sociedade atual, vem trazendo consigo oportunidades, mas também dificuldades para seus protagonistas. A população migrante corresponde a um grupo vulnerável, especialmente em problemas sanitários. A atenção ao migrante na área da saúde representa um desafio para a saúde pública, não considerando somente políticas de acesso e de informação, mas, sobretudo, pela atenção baseada num trato humanizado que respeite as crenças, valores e expectativas. Uma atenção digna exige um compromisso real dos estados e sensibilidade dos provedores de atenção dos serviços de saúde, se se pretende outorgar serviços integrais baseados no respeito dos direitos humanos a esta população.

Descritores: Migrante; Saúde; Ética.

\section{ABSTRACT}

This article aims to show that the growing phenomenon of migration in today's society has provided this community with opportunities, but also with difficulties. The migrant population is a vulnerable group, especially in sanitary matters. Health care for migrants presents a challenge for public health, not only in terms of policies regarding access and information, but most of all in matters of attention based on humanized treatment that is respectful of beliefs, values, and expectations. Dignified care demands a real commitment from the State and sensitivity on the part of health care service providers in order to offer the migrant population holistic services based on respect for their human rights.

Key words: Migrant; Health; Ethics. 


\section{INTRODUCCIÓN}

El traspaso a una sociedad globalizadora ha traído consigo una creciente interdependencia entre los pueblos, que ha facilitado a su vez la movilidad y coexistencia de culturas y razas diversas. Esta movilidad no es una novedad en tiempos contemporáneos, corresponde a un fenómeno cuyo origen es tan antiguo como el hombre mismo, y que sin lugar a dudas en las últimas décadas ha aumentado considerablemente su protagonismo. De este modo, crecientes corrientes migratorias se asocian al progreso en un mundo global, pero al mismo tiempo a aspectos negativos, o cuando menos altamente problemáticos y preocupantes.

La OIM ${ }^{(1)}$ define a las migraciones como "el movimiento de una persona o grupo de personas de una unidad geográfica hacia otra a través de una frontera administrativa o política, con la intención de establecerse de manera indefinida o temporal en un lugar distinto a su lugar de origen". Este movimiento puede obedecer a una diversidad de causas, asociadas a la búsqueda de oportunidades de trabajo, mejores condiciones socioeconómicas, continuación de estudios, huir de violaciones a los derechos humanos, traslados por desastres naturales y guerras, en definitiva se caracteriza por la búsqueda de mejores perspectivas de vida.

El Banco Mundial(2) establece que cerca de 200 millones de personas, es decir cerca del $3 \%$ de la población, vive en un país distinto al que nacieron, y esta cifra va en ascenso. En Chile, las estadísticas señalan una tendencia similar, por cuanto datos del censo 2002 arrojaban que un 1,2 \% de la población correspondían a extranjeros ${ }^{(3)}$, actualmente éstos representan a un 1,89\% de la población en territorio nacional, especialmente estos movimientos provienen de tierras latinas ${ }^{(4)}$.

La gran proporción de personas que migran constituye un proceso complejo que se traduce en riesgos y oportunidades, ofrece expectativas de mejoras en el ámbito de empleo, y un gran potencial para las economías por medio del impacto de las remesas, y muchas veces significa recuperar el respeto a los derechos humanos para quienes los ven vulnerados en sus lugares de origen. Sin embargo, también se advierte en otras oportunidades, la deshumanización asociada a este movimiento de personas, lo que se traduce en situaciones riesgosas, tanto en el traslado como en la inserción en las sociedades de destino. Los crecientes riesgos y vulnerabilidad de los migrantes representa una situación preocupante, especialmente cuando los afectados son mujeres, niños y migrantes irregulares. El fenómeno del tráfico y la trata, el tema de la discriminación, abusos y explotación, la xenofobia y la vulneración de los derechos han sido ampliamente reconocidos $^{(5)}$. Particular protagonismo han adquirido las mujeres, al aumentar paulatinamente su participación en los flujos migratorios, constituyendo actualmente casi la mitad de la población migrante en el mundo(6), en lo que se ha denominado "feminización de la migración", abriendo en algunos casos espacios para ellas y en otros perpetuando patrones de marcada desigualdad de género.

Estas situaciones han llevado a los gobiernos, organizaciones internacionales y la comunidad en general, a poner atención al impacto y a las dificultades que la población migrante puede experimentar, traduciéndose en compromisos, convenios internacionales y políticas de estado orientadas al respeto de los derechos humanos de estas personas, tales como la Convención Internacional sobre la Protección de los Derechos de todos los Trabajadores Migratorios y de sus Familiares ${ }^{(7)}$, lo que representa un esfuerzo por garantizar la promoción del bienestar e integración en las comunidades, lo que se traduce en sociedades estables y que fomentan el desarrollo en un mundo globalizado ${ }^{(8)}$.

Sin embargo, aunque se han puesto de manifiesto voluntades en atender a los problemas asociados a las migraciones, es ampliamente reconocido que las problemáticas que aquejan a la población migrante, especialmente en salud, continúan presentándose. Estos aspectos han sido analizados en países con la larga trayectoria en fenómenos de movimiento de personas, y en los que se identifican especialmente aspectos sanitarios pendientes en abordar si se pretende entregar una atención equitativa.

La salud migratoria se presenta de este modo, como un nuevo concepto especializado que se ocupa de los problemas de salud y que enfatiza el bienestar tratando las necesidades de la población migrante ${ }^{(9)}$, desde un enfoque integral. En este sentido, durante muchos años el foco de atención tradicional respecto al tema de salud de la población migrante fue tratado como un asunto de transmisibilidad de enfermedades, lo que derivó en múltiples intervenciones, tales como los programas de inmunizaciones. En la actualidad, esta sola visión representa una comprensión reduccionista de la problemática de salud de los migrantes. Se debe entender que la movilidad de la población tiene amplias repercusiones en la salud de las personas - aún cuando se trate de un proceso planificado y voluntario - enfrentándose en ocasiones a graves obstáculos para la atención sanitaria ya sea por motivos legales, culturales, idioma, discriminación, problemas económicos ó sociales hasta de desinformación. Esto representa hoy un desafío para la salud pública ${ }^{(10)}$, al enfrentarse la migración y la salud como aspectos intervinculados e interdependientes de vastas y profundas consecuencias.

\section{SALUD DEL MIGRANTE}

Según la Declaración Universal de los Derechos Huma$\operatorname{nos}^{(11)}$, toda persona, como miembro de la sociedad, tiene derecho al acceso a la seguridad social, en donde cada estado - de manera individual y mediante la cooperación internacional - debe disponer de los recursos necesarios para satisfacer las necesidades de los derechos indispensables como el de asistencia médica. De esta manera, el acceso a la atención en salud es un derecho humano universal sin distinción alguna de raza, color, sexo, idioma, religión, opinión política o de cualquier otra índole, origen nacional o social, posición económica, nacimiento o cualquier otra condición. Esta Declaración alude directamente a la población migrante al estipular claramente la no discriminación de las personas independiente de su nacionalidad, de lo que se desprende que estas personas deben recibir prestaciones sanitarias no como 
un acto de caridad, sino como un derecho humano.

El abordaje humano de la problemática migratoria en salud exige un enfoque con claros argumentos desde la perspectiva ética. Emprender la reflexión desde esta perspectiva requiere considerar que los derechos humanos se fundamentan en la dignidad de la persona. Este concepto proviene del latín dignitas, dignidad es la cualidad de digno, que hace referencia a lo correspondiente o proporcionado al merito de algo ${ }^{(12)}$. Es un valor inherente a la condición humana, es decir, está relacionado con la racionalidad y autonomía que gozan las personas. El uso de este término no se debe solamente vincular al individuo como valor innato y desvinculado de sus referencias culturales, se debe entender como un concepto complejo que debe considerar las diversidades individuales, sociales y culturales ${ }^{(13)}$.

El respeto a la dignidad de la persona es reconocer entonces al ser como un fin en sí mismo, considerando y respetando la diversidad de factores que componen su todo. Esto hace sentido en el tema del respeto a la dignidad del inmigrante, en todos los aspectos de la vida, especialmente en uno de los más vulnerables como es la salud. El sólo hecho de encontrarse enfermo en un lugar desconocido, sin las redes habituales de su país de origen, sitúan al migrante en un escenario de particular dificultad que sin duda estará asociado a las condiciones en las que como y porque se realiza el traslado, sumado a que muchas veces migra en búsqueda de la dignidad para ellos y sus familias, dignidad de la que no gozan en sus países.

Si bien los migrantes suelen encontrarse inicialmente en mejor estado de salud que los nacionales, por cuanto la salud es una ventaja que permite migrar y una condición que el país de destino controla ${ }^{(14)}$ se presentan una serie de situaciones que en mayor o menor medida vulneran la salud del migrante. Esto relacionado a que las personas que forman parte del fenómeno de movilidad sufren cambios culturales, familiares y sociales, que pueden resultar en problemas relacionados con la salud física y emocional, donde frecuentemente suele alterarse los patrones de sueño, alimentación y estados anímicos ${ }^{(15)}$, lo que en definitiva exponen a la persona a un desequilibrio en el bienestar biopsicosocial. Esto se traduce en una amplia gama de necesidades sanitarias que van desde atención en salud reproductiva, salud materno infantil, enfermedades contagiosas, intervenciones de emergencia, enfermedades crónicas, trastornos mentales y del comportamiento ${ }^{(16)}$.

Por otro lado, existen aquellas enfermedades derivadas de las condiciones laborales, donde muchas veces los trabajadores migrantes se ocupan de labores que los nacionales no quieren realizar, no solamente por sus bajos salarios, sino también asociadas a escasas condiciones de seguridad física o social que éstos ofrecen, o por su carácter forzado ${ }^{(17)}$.

Se ha documentado que la condición de migrante puede ser una importante fuente de desigualdades en salud ocupacional, entre las que se encuentran la falta de salud y entrenamiento de seguridad, el miedo a las represalias para exigir mejores condiciones laborales, barreras lingüísticas y culturales y la dificultad para acceder a la atención e indemnización cuando se lesionan ${ }^{(18)}$.
El tema de la falta del acceso información es otro punto importante a considerar, y uno de los principales obstáculos para remediar las violaciones de los derechos humanos de los migrantes ${ }^{(19)}$, ya que la falta de información en el ámbito de la salud restringe la participación de los beneficios que los estados le confieren a este colectivo de personas. Se han detectado casos en los que precisamente la falta de información de los desplazados que no están al tanto de su derecho a acceder a servicios de salud, sumado a que el personal sanitario no es consciente de su obligación de prestar estos servicios, puede disuadir a estas personas a solicitar la ayuda que necesitan ${ }^{(20)}$.

Por otro lado, los migrantes en condición irregular en términos documentarios pueden encontrarse más vulnerables a las enfermedades, por varias razones; desconocer las vías de acceso a los sistemas de salud, porque no pueden pagar la atención de salud o por temor a ser deportados. Además, frecuentemente los estados solo permiten atención en situaciones de emergencia vital para este grupo irregular ${ }^{(21)}$, lo que conduce a riesgos individuales y de salud pública ¿se daría entonces un trato digno al migrante en estas condiciones?

El trato digno en salud para el inmigrante basado en los derechos humanos se traduce en medidas de asistencia médica y de salud pública de acceso a la asistencia de salud física y mental, educación sanitaria y promoción a migrantes en general, incluyendo a los irregulares. Todos debieran tener acceso a la asistencia médica, entendiendo que se debe respetar un equilibrio entre las responsabilidades del migrante y los deberes de los Estados de acogida ${ }^{(16)}$, lo que, sin lugar a dudas, representa un tema de superior relevancia aún pendiente.

Sin lugar a dudas el trato digno en los servicios sanitarios guarda estrecha relación con los conceptos de acceso ${ }^{(22-23)}$, prevención y promoción sanitaria ${ }^{(24)}$, debe vincularse especial y estrechamente con el de trato humanizado, lo que implica otorgar prestaciones en salud en un clima amigable para los migrantes, respetando y considerando su identidad, acervo cultural, social y religioso. Para ello la Asamblea Mundial de la Salud ${ }^{(25)}$ ha solicitado a los Estados miembros que no solamente incentiven políticas de sensibilización sobre la salud de los migrantes, que promuevan el acceso equitativo a la prevención y asistencia de enfermedades para los migrantes, además de documentar y divulgar información sobre las mejores prácticas para satisfacer las necesidades de atención de salud, además de capacitar a los profesionales de la salud para hacer frente a las cuestiones de salud relacionadas con la migración de poblaciones. Ciertamente el aumento de la migración en el mundo enfrenta al personal sanitario cada vez más a usuarios de diferentes orígenes étnicos y culturales. Lo que no se traduce solamente en la variación de las problemáticas de salud, sino en las diferentes necesidades y expectativas en la atención $^{(26)}$. Estas diferencias culturales deben tenerse presente por cuanto influyen en las expectativas de las personas ${ }^{(27)}$. Por tanto, la labor de los profesionales sanitarios debe ser sensible a la diversidad cultural de las poblaciones a las que presta sus servicios, y aplicar sus conocimientos científicos adaptados a esa pluralidad cultural siendo receptivos en el quehacer ${ }^{(28-29)}$. Es en este punto donde el concepto interculturalidad en salud se presenta abarcando y considerando la atención sanitaria 
dirigida a personas provenientes de latitudes distintas, como un proceso estratégico, dinámico y permanente de comunicación y aprendizaje entre culturas, basados en un contacto de respeto ${ }^{30}$. Esta interculturalidad no sólo debe estar presente en las políticas sanitarias, sino también y especialmente en la formación de los proveedores de salud quienes son los que en definitiva entregan la atención sanitaria a esta población.

Los agentes de salud deben ser capaces de desenvolverse en esa diversidad. Entender a las personas de manera holística: sus problemas físicos, emocionales y sociales, su pasado y su futuro, y las realidades del mundo en que viven, es decir, el trabajador sanitario debe comprender los distintos casos y situaciones que enfrentan las personas, lamentablemente a veces los proveedores de atención descuidan este aspecto, sobre todo cuando atienden a grupos más desfavorecidos ${ }^{(31)}$ como la población migrante.

Actualmente es reconocida la importancia de la participación activa de los usuarios en salud, lo que exige también un enfoque integral en la atención sanitaria, la que se traduce en la consideración y entendimiento de los valores y percepciones del paciente. Ha sido largamente documentada la importancia creciente en la participación del paciente en salud. La influencia de la cultura y del idioma influyen en la participación y compromiso de la persona en salud, contribuyendo a reducir la incidencia y el impacto de sucesos adversos evitables en la atención de la salud ${ }^{(32-33)}$. En este sentido, las creencias personales determinan conductas preventivas y de adhesión en salud y que una mayor participación en la sociedad de acogida es concomitante a un aumento del uso de los servicios de salud ${ }^{(34)}$

En este contexto si las sociedades aspiran otorgar prestaciones sanitarias basadas en los derechos conferidos y los convenios establecidos, se debe considerar una atención digna al migrante como una necesidad de primera línea en salud, que exige en la práctica la participación de aspectos culturales y las necesidades particulares de esta población.

\section{CONSIDERACIONES FINALES}

La población migrante constituye un grupo vulnerable en salud, al presentarse una serie de variables asociadas con el fenómeno de traslado y asentamiento que pueden alterar el equilibrio biopsicosocial. Se han manifestado intenciones claras de resguardar el derecho a salud de esta población, empero continúan claras situaciones de desigualdad en la atención sanitaria. No solamente en la información, acceso, sino que aparejado a ello una atención que debe dar énfasis especialmente a una atención humanizada, considerando el acervo cultural, creencias, idioma, valores y expectativas, en definitiva un trato digno al migrante, independiente de su condición.

La atención sanitaria humanizada al migrante se presenta entonces como una tarea que no sólo exige presencia en la agenda internacional, sino que un compromiso real de los estados y sensibilidad de los proveedores de atención de los servicios de salud, si se pretende otorgar servicios integrales basados en el respeto de los derechos humanos.

\section{REFERENCIAS}

1. OIM. Tipologías de la Migración. [citado el 20 Marzo 2010]. Disponible en: http://www.iom.int/jahia/Jahia/ about-migration/migration-management-foundations/ terminology/migration-typologies/cache/offonce/lang/es

2. Banco Mundial. Migraciones y remesas. 2008. [citado el 20 Marzo 2010]. Disponible en: http://www.bancomundial.org/temas/remesas/

3. INE. Censo 2002 Síntesis de Resultados. [citado el 20 Marzo 2010]. Disponible en http://www.ine.cl/cd2002/ sintesiscensal.pdf

4. Departamento De Extranjería y Migración Ministerio Del Interior. Informe Anual Estimación de Extranjeros en Chile. 2010

5. CEPAL. Migración internacional, derechos humanos y desarrollo en América latina y el caribe síntesis y conclusiones. 2006

6. OIM. Migración y género. 2008. [citado 11 de Diciembre 2008]. Disponible en: http://www.oim.int

7. ONU. Convención internacional derechos de los trabajadores migratorios y sus familias 1990; [citado 05 de Marzo 2010]. Disponible en: http://www.unhchr.ch/tbs/doc. nsf/c12563e7005d936d4125611e00445ea9/a0d5b166e c404486c1256fe0004ad28a/\$FILE/G0540821.pdf
8. OIM. Migración y salud para beneficio de todos. Octogésima reunión 2004; [citado 11 de Marzo 2010]. Disponible en: http://202.173.4.113/jahia/webdav/shared/shared/ mainsite/about_iom/es/council/88/MC_INF_275_sp.PDF

9. OIM. Migración y salud. 2009. [citado el 10 de Junio 2009] Disponible en: http://www.iom.int/jahia/Jahia/about-iom/ organizational-structure/migration-health-department/ lang/es

10. OMS. Migración Internacional, Salud y Derechos Humanos 2003; [citado 10 de Abril 2009] Disponible en: http:// www.who.int/hhr/activities/2005\%20PRT\%2016325\%20 ADD\%201\%20Migr_HHR-Spanish\%20edition.pdf

11. ONU. Declaración universal de los Derechos Humanos 1948; [citado 06 de Marzo 2010] Disponible en: http:// www.unhchr.ch/udhr/lang/spn.htm

12. Real Academia Española. Diccionario de la lengua española. 22 a ed. Madrid : Espasa-Calpe, 2006.

13. Pyrrho M, Cornelli G, Garrafa V. Dignidad humana. Reconocimiento y operacionalización del concepto. Acta Bioética. 2009; 15(1): 65-69

14. United Nations Population Fund. Estado de la Población Mundial 2006, "Hacia la Esperanza, las mujeres y la migración internacional". 2006. 
15. Moya J. y Uribe M. Migración y Salud en México: Una aproximación a las perspectivas de investigación; 19962006. 2006. [citado 10 de Marzo 2010]. Disponible en:http://www.mex.ops-oms.org/contenido/migracion.htm.

16. OIM. Migrantes Particularmente Vulnerables. . [citado 13 de Marzo 2010]. Disponible en: http://www.iom.int/ jahia/Jahia/about-migration/developing-migration-policy/ migration-and-health/particularly-vulnerable-migrants/ lang/es45\%20https://apps.who.int/gb/ebwha/pdf_files/ A61/A61_R17-sp.pdf.

17. OIT. La OIT y los trabajadores migrantes . 2006 [citado 13 de Marzo 2010]. Disponible en. http://www.oit.org.pe/index.php?option $=$ com_content\&view $=$ article\&id $=295$ :laoit-y-los-trabajadores-migrantes\&catid = 114:especiales\&lt emid $=374$.

18. Patino C. Kirchner T. Stress and Psychopathology in LatinAmerican Immigrants: The Role of Coping Strategies. Psychopathology . 2010; 43(1): 17- 24.

19. Pizarro J. El mapa migratorio de América Latina y el Caribe, las mujeres y el género. Serie Población y Desarrollo CEPAL. 2003.

20. OIM. 06-03-2009 La Vulnerabilidad de las Migrantes se ve Exacerbada porque no Tienen Acceso a Cuidados de Salud Maternoinfantil. 2009. [citado 13 de Marzo 2010]. Disponible en. http://202.173.4.113/jahia/Jahia/media/ news-releases/newsArticleEU/cache/offonce/lang/es;jse ssionid = 36FD5CF6C577A7FF4D63744485B6C1DA worker02? entryld $=23934$.

21. Carrasco D. Informe global de buenas prácticas en inmigración e instituciones nacionales de derechos humanos. 2007.

22. Amon J. , Todrys K. ne. Acceso a tratamiento antirretroviral para las poblaciones migrantes del sur Global. Sur, Rev. int. direitos human. 2009; 6 (10): 162 - 187.

23. Yi HS, Mantell JE, Wu RR, et al .A profile of HIV risk factors in the context of sex work environments among migrant female sex workers in Beijing, China. Psychology Health \& Medicine. 2010; 15 (2): 172-187 [citado 13 de Marzo 2010]. Disponible en http://www.informaworld.com/smpp/ content $\sim$ content $=$ a920248700 $\sim \mathrm{db}=$ all $\sim$ jumptype $=$ rss
24. Spallek J, Zeeb H, Razum O. Prevention among immigrants: the example of Germany. BMC public health . 2010; 10 (92). [citado 13 de Marzo 2010]. Disponible en http://www.biomedcentral.com/1471-2458/10/92

25. OMS. $61^{\text {a }}$ Asamblea Mundial De La Salud. 2008

26. Harmsen J A, Bernsen RM, Bruijnzeels M A, Meeuwesen L. Patients' evaluation of quality of care in general practice: What are the cultural and linguistic barriers? Patient Education and Counseling 2008; 72: 155-162.

27. Clemens MD. En: Harmsen J A, Bernsen RM, Bruijnzeels M A, Meeuwesen L. Patients' evaluation of quality of care in general practice: What are the cultural and linguistic barriers? Patient Education and Counseling 2008; 72:155-162

28. González T, Martínez L, Luengo R. Antropología de los cuidados en el ámbito académico de la enfermería en España. Texto contexto - enferm. [serial on the Internet] 2006; [citado el 14 de Mayo]; 15(1): 155-161. Disponible desde: http://www.scielo.br/scielo.php?script =sci arttext\&pid = S0104-07072006000100020\&Ing = en.

29. Pinson AL. Enfermería y diversidad cultural: una mirada desde las Américas. Index de Enfermería 2003; XII (43): 40-4

30. Sáez M. Interculturalidad En Salud En Chile: De La Teoría A La Práctica

31. OMS. Informe sobre la salud en el mundo 2008 - La Atención primaria de salud, más necesaria que nunca. 2008.

32. Johnstone M. Kanitsaki O. Culture, Language, And Patient Safety:Making The Link .International Journal For Quality In Health Care 2006; 1- 6

33. Charles C. , Gafni A., Whelan T., O'Brien M. Cultural influences on the physician-patient encounter: The case of shared treatment decision-making. Patient Education and Counselin. 2006. 63: 262-267.

34. Fassaert $T$, Hesselink AE, Verhoeff AP.Acculturation and use of health care services by Turkish and Moroccan migrants: a cross-sectional population-based study Bmc Public Health [citado 18 de Abril 2010]. Disponible en: http://www.biomedcentral.com/1471-2458/9/332 